\title{
List of Reviewers for this Special Issue
}

Journal of International Business Studies (2008) 39, 705. doi: 10.1057/palgrave.jibs.8400374

The guest editors wish to acknowledge and thank members of the Editorial Review Board and the scholars listed below who have contributed their time to review manuscripts for this "Institutions and International Business" Special Issue.

Adler, Paul

Aguilera, Ruth

Ahmidjian, Christina

Alcacer, Juan

Beamish, Paul

Benito, Gabriel

Berry, Heather

Boddewyn, Jean

Boyacigiller, Nakiye

Brown, Dana

Clougherty, Joseph

Coeurderoy, Regis

Cosset, Jean-Claude

Cuervo-Cazurra, Alvaro

Deeg, Richard

Delios, Andrew

Doh, Jonathan

Dunning, John H

Fey, Carl

Fisman, Ray

Fiss, Peer

Foley, C Fritz

Guillén, Mauro F

Guler, Isin
Holburn, Guy

Husted, Bryan

Jandhyala, Srividya

Jones, Geoff

Jovorcik, Beata Smarzynska

Kobrin, Steve

Kostova, Tatiana

Leca, Bernard

Lessard, Don

Luo, Xiaoqu

MacDuffie, John Paul

Mathur, Ike

Mayer, Kyle

McDermott, Gerald

Meyer, Klaus

Miller, Stewart

Morgan, Glenn

Oviatt, Ben M

Perkins, Susan

Poppo, Laura

Prakash, Aseem

Ramamurti, Ravi

Reisel, William
Ricart, Joan-Enric

Roehl, Tom

Salomon, Robert

Santarelli, Enrico

Siegel, Jordan

Somaya, Deepak

Spencer, Jennifer

Steensma, Kevin

Subramaniam, Mohan

Teng, Bing-Sheng

Tschoegl, Adrian

Westney, Eleanor

Wezel, Filippo Carlo

White, George

Wijen, Frank

Wilkins, Mira

Woywode, Michael

Wright, Mike

Zander, Lena

Zelner, Bennet 